\title{
Taiwan als ständiger Beobachter bei der Weltgesundheitsorganisation (WHO)?
}

\author{
Von Rainer Lagoni, Hamburg
}

\section{Gesundheit als globale Aufgabe}

Seit dem Ende des Zweiten Weltkriegs ist die durchschnittliche Lebenserwartung der Bevölkerung in vielen Ländern statistisch nachweisbar gestiegen. Gründe für diese Entwicklung liegen nicht zuletzt in einer verbesserten Medizin, Pharmakologie und Hygiene. Und doch erlebt die Menschheit im Bereich der Gesundheit immer wieder regionale Epidemien, die weltweit in Pandemien umzuschlagen drohen. Die grenzüberschreitende Natur dieser Gefahren stellt die Gesundheitsverwaltungen vor immer neue Herausforderungen und macht ihre internationale Zusammenarbeit unverzichtbar.

Das wichtigste internationale Forum für diese Zusammenarbeit im Bereich der Gesundheit ist die am 7. April 1948 als Sonderorganisation der Vereinten Nationen errichtete Weltgesundheitsorganisation (WHO). Sie hat heute 192 Mitgliedstaaten und repräsentiert damit die gesamte Staatengemeinschaft. Ihr allgemeines Organ ist die Weltgesundheitsversammlung (World Health Assembly, WHA), die über das Programm, das Budget und die Ausrichtung der Politik der Organisation entscheidet. Wichtige operative Entscheidungen der WHO trifft ferner der Exekutivrat; die Verwaltung liegt in den Händen des Generaldirektors, dem ein Sekretariat zur Seite steht.

Die Aufgabe der WHO besteht in der Erreichung eines möglichst hohen Gesundheitsstandards für alle Völker. Gesundheit wird dabei umfassend verstanden. Sie besteht nicht nur in der Abwesenheit von Krankheit oder Gebrechen, sondern ist ein Zustand des völligen körperlichen, psychischen und sozialen Wohlbefindens. ${ }^{1}$ Entsprechend ihrem Auftrag kümmert sich die WHO unter anderem um die Bekämpfung von Aids und Malaria sowie um die Vorbeugung von Bioterrorismus, aber auch um die Biotechnologie und die Bekämpfung des Rauchens. ${ }^{2}$ Seit dem Ausbruch der so genannten Vogelgrippe gehören zu

Vgl. die Präambel der Satzung der Weltgesundheitsorganisation vom 22.7.1946, in Kraft für die Bundesrepublik Deutschland seit dem 29.5.1951, BGBl. 1974 II 43, UNTS Bd. 14, S. 185, Bd. 377, S. 380. de la santé, Paris 1995; Michel Bélanger, Droit international de la santé, Paris 1983; Gerald G. 
ihren aktuellen Aufgaben insbesondere auch die Fälle menschlicher Erkrankungen an dem H5N1-Virus, indem sie auf internationaler Ebene Maßnahmen koordiniert und Vorbereitungen trifft, um eine weltweite Verbreitung dieser Krankheit zu verhindern.

Taiwan gehört der WHO nicht als Mitglied an, dies entspricht der Satzung der Organisation, nach der nur Staaten Mitglieder der WHO sein können. Angesichts der erwähnten Lage der Weltgesundheit und der weltumspannenden Aufgaben der WHO stellt sich aber die Frage, ob die Gesundheitsverwaltung Taiwans als eine „Einheit im Gesundheitswesen“ (health entity) anzusehen ist, die einen Status als ständiger Beobachter bei der WHO genieBen sollte. Als Beobachter hätte die Taiwan Health Entity nicht die gleichen Rechte wie die Mitglieder der Organisation. Sie könnte zwar an den Sitzungen der Weltgesundheitsversammlung teilnehmen und sich dort auch mit eigenen Vorschlägen an den Debatten beteiligen, so dass sie insbesondere am Informationsfluss teilnehmen würde. Als Beobachter hätte sie aber kein Stimmrecht und wäre auch nicht an der Feststellung eines Konsenses beteiligt, so dass sie keinen unmittelbaren Einfluss auf Entscheidungen der Versammlung zur Gesundheitspolitik nehmen könnte. Derzeit gibt es sechs Beobachter bei den Sitzungen der Weltgesundheitsversammlung. Dies sind der Heilige Stuhl, Palästina, der Souveräne Malteser-Ritterorden, das Internationale Komitee vom Roten Kreuz (IKRK), die Internationale Föderation der Rotkreuz- und Rothalbmondgesellschaften sowie die Interparlamentarische Union (IPU). ${ }^{3}$

Eine „Einheit im Gesundheitswesen“ (health entity) hat indes bisher noch keinen Beobachterstatus bei der WHO inne. Jedoch gibt es in anderen völkerrechtlichen Bereichen als dem Gesundheitswesen Beispiele, in denen auf pragmatische Weise eine sachlich gebotene Beteiligung Taiwans ermöglicht worden ist, ohne dass der Status der Insel dadurch berührt würde. So ist Taiwan unter dem weltweiten sog. Straddling Stocks Übereinkommen von $1995^{4}$ und einem entsprechenden Fischereiübereinkommen für den westlichen und mittleren Pazifik ${ }^{5}$ als , fishing entity“ anerkannt, ohne formell Vertragspartei dieser völkerrechtli-

Sander, Internationaler und europäischer Gesundheitsschutz, Baden-Baden 2004; Javed Siddiqi, World health and world politics: the World Health Organization and the UN system, London 1995.

3 Die rechtlichen Grundlagen für deren Beobachterstatus werden im Einzelnen an späterer Stelle analysiert.

4

Übereinkommen vom 4.8.1995 zur Durchführung der Bestimmungen des Seerechtsübereinkommens der Vereinten Nationen vom 10.12.1982 über die Erhaltung und Bewirtschaftung von gebietsübergreifenden Fischbeständen und Beständen weit wandernder Fische, Art: 1.3, 9.2 und 17.3 (BGBl. 2000 II 1022).

Convention on the Conservation and Management of Highly Migratory Fish Stocks in the Western and Central Pacific Ocean 2000, Art. 9.2 und Annex I (Int. Legal Materials, Bd. 40 (2001), S. 278). 
chen Verträge zu sein. Diese werden nicht nur auf Taiwan entsprechend angewendet; ${ }^{6}$ als eine anerkannte Verwaltungseinheit hat Taiwan in beiden Fällen einen Beobachterstatus in den Fischereiorganisationen inne und ist voll in die Durchführung der Verträge einbezogen. In einem ähnlich pragmatischen Geist ist die Welthandelsorganisation (WTO) noch weiter gegangen, indem sie im November 2001 dem Beitritt der Zollterritorien von Taiwan, Penghu, Kinmen und Matsu als „Chinese Taipei“ zugestimmt hat.

Indes geht die hier in Frage stehende Teilnahme der Gesundheitsverwaltung von Taiwan als Beobachter an den Sitzungen der WHO offensichtlich weniger weit als Taiwans Beteiligung in den erwähnten Fällen. Gleichwohl wirft sie eine Reihe völkerrechtlicher und politischer Fragen auf: Wie weit reicht diese Teilnahme; könnte eine ähnlich pragmatische Lösung im Rahmen der WHO gefunden werden; besteht neben der sachlichen Notwendigkeit auch eine rechtliche Verpflichtung der WHO zur Aufnahme der Taiwan Health Entity als Beobachter? Und schließlich ist auch zu fragen, ob ein derartiger Beobachterstatus mit den UN Resolutionen hinsichtlich der Repräsentation Chinas zu vereinbaren wäre und er die One China Policy berühren würde.

\section{Beobachterstatus und Satzung der WHO}

Wie jede andere zwischenstaatliche internationale Organisation regelt die WHO ihre internen rechtlichen Angelegenheiten und ihre auswärtigen Beziehungen auf der Grundlage ihres Gründungsvertrages selbst. Ihre Satzung vom 22.7.1946 behandelt neben der Aufnahme neuer Mitglieder ${ }^{8}$ auch eine assoziierte Mitgliedschaft. Diese steht Territorien oder Gruppen von Territorien offen, die nicht für die Durchführung ihrer internationalen Beziehungen verantwortlich sind. ${ }^{9}$ Zudem enthält sie Bestimmungen über die Beziehungen zu den Vereinten Nationen und zu anderen zwischenstaatlichen Organisationen. ${ }^{10}$ Die Konsultation von nichtstaatlichen Organisationen (NGOs) und Vereinbarungen über eine Kooperation mit ihnen sowie mit staatlichen oder privaten nationalen Organisationen sind in Artikel 71 geregelt. ${ }^{11}$ Nirgendwo jedoch sieht die WHO-Satzung einen Beobachterstatus

6

8

10

11

Art. 1 Abs. 3 Straddling Stocks Übereinkommen, siehe Anm. 4.

WTO, Dokument Nr. WT/L/433 vom 23. November 2001.

Mitgliedschaft in der WHO steht allen Staaten offen, unabhängig davon, ob diese Mitglieder der UNO sind, Artikel 3, 4 und 6 der Satzung der WHO.

Artikel 8 der Satzung der WHO.

Artikel 69, 70 der Satzung der WHO.

Darüber hinaus hat die 40. Weltgesundheitsversammlung Regelungen hinsichtlich der Zusammenarbeit mit NGOs erlassen, die neben informellen ad hoc Kontakten und Arbeitsbeziehungen auch die Etablierung offizieller Beziehungen vorsehen (WHA, Principles Governing Relations between the World Health Organization and Nongovernmental Organizations, Dokument Nr. WHA40.25). 
vor. Auch wird der Begriff einer „Einheit im Gesundheitswesen“ (health entity) von ihr nicht verwendet oder in einem Dokument der Organisation förmlich anerkannt.

Die Möglichkeit einer Teilnahme jeglicher Organisation, die Gesundheitsaufgaben innehat, ist zwar ausdrücklich in der Satzung der WHO vorgesehen. ${ }^{12}$ Angesichts des Mangels an weiteren Bestimmungen der Satzung wäre in Bezug auf Taiwan aber einzig zu erwägen, ob eine Einheit im Gesundheitswesen eine ,nationale Organisation“ im Sinne des Artikels 71 der Satzung darstellen könnte. Dagegen spricht jedoch, dass der Begriff einer ,nationalen Organisation" auf die Staatlichkeit der Herkunftsnation abstellt und daher der besonderen Situation Taiwans gerade nicht gerecht wird. Überdies könnte die Einladung einer taiwanesischen Health Entity zur Teilnahme an den Sitzungen der Weltgesundheitsversammlung auf dieser Rechtsgrundlage zu Rückschlüssen hinsichtlich der Rechtslage Taiwans führen, die nicht erwünscht wären. Außerdem setzt jegliche Einladung die Zustimmung der Regierung des betroffenen Vertragsstaates voraus, die von Taiwan nicht zu erbringen wäre. Schließlich würde die Stellung als nationale Organisation den praktischen Bedürfnissen dauerhafter Beziehungen schwerlich gerecht werden, weil eine derartige Einladung jeweils erneuert werden müsste. Ein ständiger Beobachterstatus einer Health Entity, auch wenn dieser nicht ausdrücklich in der Satzung geregelt ist, hätte demgegenüber den Vorteil einer unpolitischen Institutionalisierung der Beziehungen.

\section{Derzeit zugelassene Beobachter}

Die WHO-Satzung stellt indessen nur einen rechtlichen Rahmen dar, in dem die Weltgesundheitsversammlung unter anderem ihre eigene Verfahrensordnung erlässt. ${ }^{13}$ Diese sieht im Unterschied zur Satzung auch einen Beobachterstatus bei der Weltgesundheitsversammlung vor. Allerdings soll dieser Beobachterstatus allein ein Vorstadium zur Aufnahme als Mitglied oder als assoziiertes Mitglied der WHO sein. ${ }^{14}$ Dies hatte in einer früheren Zeit, als viele Staaten noch kein Mitglied und zahlreiche Territorien noch nicht unabhängig waren, durchaus einen Sinn. Ihnen wurde damit ein vorläufiger, auf die spätere Mitgliedschaft in der Organisation gerichteter Status geboten. Ein derartiger Beobachterstatus

Artikel 18(h) der Satzung der WHO.

Artikel 17 der Satzung der WHO.

14 Vgl. Regel 3 Abs. 2, Regel 19 der Verfahrensordnung der Weltgesundheitsversammlung. Die Regel wurde von der Achten Weltgesundheitsversammlung beschlossen und ist in ihrer gegenwärtigen Fassung einsehbar unter <http:/www.who.int/governance/en>. Darüber hinaus können auch Staaten, welche die Satzung der WHO unterzeichnet aber nicht ratifiziert haben, einen Beobachterstatus erhalten. Die Rechte der Beobachter sind in Regel 47 festgelegt. Überdies können zwischenstaatliche oder nichtstaatliche Organisationen den Status eines ,eingeladenen Vertreters“ beantragen und auf diese Weise an den Versammlungen teilnehmen. 
scheidet aber aufgrund der besonderen, eine Mitgliedschaft ausschließenden Rechtslage Taiwans aus.

Die gegenwärtig sechs ständigen Beobachter ${ }^{15}$ bei der Weltgesundheitsversammlung haben jedoch allesamt ihren Status nicht aufgrund dieser Regelungen der Verfahrensordnung erhalten. Keiner von ihnen befindet sich im Vorstadium einer Mitgliedschaft bei der WHO, denn keiner erfüllt die Anforderungen an einen Staat, so dass eine solche für sie ausscheidet. ${ }^{16}$ Dennoch wurde ihnen - weder eindeutig auf die Satzung noch auf die Verfahrensordnung gegründet - ein ständiger Beobachterstatus eingeräumt.

Es zeigt sich demnach, dass auch die Verfahrensordnung insoweit nicht abschließend ist und somit weitere Möglichkeiten, einen ständigen Beobachterstatus zu gewähren, bestehen. Ein Beleg für eine derartige Möglichkeit findet sich schon in der WHO-Satzung selbst. Diese räumt der Weltgesundheitsversammlung das Recht ein, jegliche weitere Handlung vorzunehmen, die angemessen ist, um den Zweck der Organisation zu erfüllen. ${ }^{17}$ Dies entspricht dem allgemeinen Rechtsgrundsatz, dass ohnehin jeder internationalen Organisation die Befugnisse zustehen, die für die Erreichung ihrer satzungsmäßigen Ziele wesentlich sind, auch wenn sie in ihrer Satzung bzw. ihrem Gründungsvertrag nicht ausdrücklich erwähnt sind. ${ }^{18}$

Außerdem könnte man auch daran denken, dass sich mit der Aufnahme der sechs Beobachter bereits eine diesbezügliche Praxis etabliert hat. ${ }^{19}$ Bei keiner der Einladungen wurde

Der Heilige Stuhl, Palästina, der Souveräne Malteser-Ritterorden, das Internationale Komitee vom Roten Kreuz (IKRK), die Internationale Föderation der Rotkreuz- und Rothalbmondgesellschaften und die Interparlamentarische Union (IPU).

Beim IKRK, der Internationalen Föderation der Rotkreuz- und Rothalbmondgesellschaften und der IPU ist der Mangel des Charakters als souveräner Staat offensichtlich, aber auch beim Heiligen Stuhl, Palästina und dem souveränen Malteser-Ritterorden handelt es sich nicht um Staaten, sondern um allenfalls Völkerrechtssubjekte sui generis, da ihnen zumindest eins der Staatselemente fehlt; vgl. Volker Epping, in: Ipsen, Völkerrecht, 5. Aufl., München 2004, S. 100 ff.; Alfred Verdross / Bruno Simma, Universelles Völkerrecht, 3. Aufl., Berlin 1984, S. 247 ff., 252, 253 ff.

Artikel 18(m) der Satzung der WHO.

18 Vgl. den Internationalen Gerichtshof, Reparations for Injuries Suffered in the Service of the United Nations, I.C.J. Rep. 1949, S. 174, 182.

19

Der Heilige Stuhl wurde bereits 1949 zum ersten Mal und seit 1953 in jährlicher Folge als Beobachter eingeladen. Die Einladung des souveränen Malteserordens wurde 1963 zum ersten Mal durch den Generaldirektor ausgesprochen und seitdem jährlich erneuert. Im Hinblick auf Palästina gab es zunächst eine Resolution der Weltgesundheitsversammlung zur Einladung von Vertretern nationaler Befreiungsbewegungen, die von der Organisation für Afrikanische Einheit (OAU) oder der Liga Arabischer Staaten anerkannt waren, als Beobachter (Resolution WHA27.37 vom 21. Mai 1974). Einzig Palästina wurde aufgrund dieser Resolution eingeladen. Später wurden dann die Rechtsbeziehungen zu Palästina neu gefasst und durch die Resolution WHA53.13 vom 20. Mai 2000 erheblich erweitert. Sowohl das IKRK als auch die Internationale Föderation der Rot- 
indes die Rechtsgrundlage des Handelns dargelegt. Auch bleibt offen, wer das Recht zur Einladung hat. Es scheint sich jedoch das Verfahren herausgebildet zu haben, dass die Entscheidung zur Einladung vom Exekutivrat der WHO auf Ersuchen des jeweiligen Interessenten hin getroffen wird. Der Exekutivrat ermächtigt daraufhin den Generaldirektor, die Einladung auszusprechen.

Die Praxis der WHO hinsichtlich ihrer bisherigen ständigen Beobachter zeigt also, dass die Organisation selber eine pragmatische Lösung der anfallenden Probleme befürwortet. Entsprechend ihren Zielen realisiert sie die Notwendigkeit, im internationalen Gesundheitswesen involvierten nichtstaatlichen Rechtssubjekten möglichst umfassend die Möglichkeit einer Teilnahme an den Sitzungen der Weltgesundheitsversammlung zu gewähren. Dass diese Notwendigkeit auch in Bezug auf Taiwan besteht, liegt nicht erst seit der Möglichkeit einer durch das H5N1-Virus ausgelösten Pandemie auf der Hand.

\section{Verpflichtung der WHO zur Institutionalisierung der Beziehungen}

Die Bedeutung der WHO für die Gesundheit der Weltbevölkerung spiegelt sich in ihrem universellen Auftrag wieder. In diesem Sinne bestimmt die Präambel ihrer Satzung, dass der Genuss einer möglichst guten Gesundheit ein Grundrecht jedes Menschen ist, das ohne Unterschied der Rasse, der Religion, der politischen Überzeugungen oder der wirtschaftlichen oder sozialen Lage besteht. In diesem Sinne bestimmt auch Artikel 1 der WHOSatzung, dass das Ziel der Organisation die Erreichung eines möglichst guten Gesundheitszustands für alle Völker ist. Nun kann im Falle von Taiwan zwar nicht von einem Volk, sondern nur von einer Bevölkerung gesprochen werden. Gleichwohl gebietet es die in der Präambel erwähnte menschenrechtliche Natur der Gesundheit, dass das Ziel eines möglichst guten Gesundheitszustands für die Bevölkerung von Taiwan entsprechend gelten muss.

Ein weiterer Ausdruck des satzungsgemäßen Zieles der Organisation ist ihre Verpflichtung zur effektiven Zusammenarbeit mit auf Gesundheit spezialisierten Agenturen, nationalen Gesundheitsbehörden, Berufsgruppen, nichtstaatlichen und sonstigen Organisationen, die

kreuz und Rothalbmondgesellschaften und die IPU hatten als NGO zunächst offizielle Beziehungen mit der WHO, die später durch den Exekutivrat der WHO zum Status eines Beobachters aufgewertet wurden (vgl. EB101.R21 vom 27. Januar 1998, EB103.R18 vom 1. Februar 1999 und EB113.R14 vom 23. Januar 2004). Die offiziellen Einladungen dieser beiden NGOs wurden dann durch den Generaldirektor ausgesprochen. Einzelheiten finden sich in der unveröffentlichten Stellungnahme von Sir Arthur Watts, Preliminary Opinion on „The Legality of Taiwan's Participation in the World Health Assembly as an Observer in the Capacity of a Health Entity", London, 24. April 2005, 23 S. 
dazu geeignet erscheinen. ${ }^{20}$ Die WHO hat also umfassende Möglichkeiten, mit praktisch sämtlichen Akteuren des Gesundheitsschutzes zusammenzuarbeiten. Die assoziierte Mitgliedschaft von Gebieten, die nicht eigenständig für ihre internationalen Beziehungen verantwortlich sind, macht ebenso ihren Auftrag deutlich, entsprechend ihrem Ziel an der Verbesserung der Gesundheit aller Völker und Bevölkerungen zu arbeiten. Eine im Lichte ihres Auftrags restriktive und streng formalistische Auslegung ihrer Satzung oder Verfahrensordnung wäre nicht als angemessen zu bezeichnen. Die Organisation ist dazu verpflichtet, sämtliche notwendigen Handlungen zu unternehmen, die zur Erreichung ihres Zieles erforderlich sind. ${ }^{21}$ Dieses Ziel begreift sie selbst umfassend, wie die geschilderte pragmatische Aufnahme von sechs ständigen Beobachtern zeigt. Daran muss sie sich messen lassen.

Vor diesem rechtlichen Hintergrund ist die Notwendigkeit zur Einbeziehung der taiwanesischen Gesundheitsverwaltung in die Verhandlungen der Weltgesundheitsversammlung näher darzulegen: Der Schutz der 23 Millionen Bewohner Taiwans erfordert einen ständigen und schnellen Informationsaustausch zwischen den Gesundheitsbehörden zum Schutz vor Epidemien, wie sie etwa durch das H5N1-Virus drohen könnten, oder etwa im Fall von SARS $^{22}$ gedroht haben. Generell ist nicht davon auszugehen, dass die Behörden der Volksrepublik China in solchen Situationen die notwendigen Informationen über die Gesundheitssituation in Taiwan haben. ${ }^{23}$ Eine faktische Vertretung Taiwans durch China in der Weltgesundheitsversammlung ist daher gerade in schwierigen Lagen nicht möglich. Ein effektiver Schutz der 23 Millionen Bewohner Taiwans durch die WHO wird allein durch eine Beteiligung einer Taiwan Health Entity gewährleistet, die auch sämtliche Informationen über dortige Gesundheitsrisiken und -erkrankungen der Bevölkerung besitzt.

Überdies erfordert nicht nur der Schutz der dortigen Bevölkerung einen ständigen Beobachterstatus bei der WHO. Auch der Schutz der Weltbevölkerung macht eine wirksame

Artikel 2(b) der Satzung der WHO.

21

Artikel 2(v), 18(m) der Satzung der WHO.

22 Severe Acute Respiratory Syndrome, Schweres akutes Atemnotsyndrom. Nach dem Ausbruch dieser Krankheit hat die WHO Experten nach Taiwan zum Bericht über SARS geschickt, siehe Dokumente EB114/2004/REC/1 und EB113/33 des Exekutivrates.

23

Nach Angaben des Generaldirektors der WHO gibt es ein Memorandum of Understandig zwischen der Volksrepublik China und der WHO über einen Mechanismus, aufgrund dessen Taiwanesische Experten an den technischen Aktivitäten der WHO teilnehmen können und Mitarbeiter der WHO dorthin im Falle eines Notfalls entsandt werden können (vgl. Dokument EB116/2005/REC/1 des Exekutivrates). Danach muss jedoch für jeden einzelnen Fall eine Einwilligung der Behörden der Volksrepublik eingeholt werden. Die Gesundheit der Bevölkerung Taiwans ist daher bislang von dem Wohlwollen, der Schnelligkeit des Informationsflusses und der Entscheidungsbildung auf Seiten der Volksrepublik China sowie von der aktuellen politischen Lage auf beiden Seiten der Taiwan-Straße abhängig. 
Einbeziehung der Bewohner Taiwans in das System der Organisation notwendig. Die WHO weist selbst in ihrer Resolution WHA54.14, "Global health security: epidemic alert and response" darauf hin, dass eine effektive Seuchenbekämpfung nur möglich sei, wenn diese alle Völker der Welt umfasst. Derzeit ist Taiwan jedoch nicht dem Internationalen Seuchenvorwarnungs- und Seuchenbekämpfungsnetz (Global Outbreak Alert and Response Network, GOARN) angeschlossen und gehört zahlreichen anderen Überwachungs- und Informationsnetzwerken nicht an. ${ }^{24}$ Auch verweigert etwa das Regionalbüro der WHO für den Westpazifik bisher den Austausch über Seuchenvermeidungspläne mit Taiwan. Zusätzlich wird Taiwan die Teilnahme an formellen und informellen Treffen der WHO verweigert.

Dies kann sich als gefährlich erweisen, denn Taiwan ist ein bedeutender Verkehrsknotenpunkt im westlichen Pazifik. Es ist Heimat für etwa 315.000 Gastarbeiter aus südostasiatischen Ländern und etwa 10.000 Bürger der EU. Ferner verzeichnet die Insel jährlich mehr als eine halbe Million Besucher aus südostasiatischen sowie etwa 180.000 Besucher aus europäischen Ländern, darunter 39.000 Deutsche. ${ }^{25}$ Im Jahre 2004 besuchten zudem mehr als 1,43 Millionen Reisende aus Taiwan südostasiatische Länder. In Zeiten der sog. Vogelgrippe ist auch nicht außer Betracht zu lassen, dass jährlich etwa 1,25 Millionen Zugvögel, 351 unterschiedliche Arten, auf ihrem Weg von Sibirien und China über Japan und Korea zu den Philippinen und nach Indonesien, Malaysia oder Australien in Taiwan Station machen.

Im Ergebnis zeigen diese Gefahren für die Weltbevölkerung, dass die WHO zur Erfüllung ihres Auftrags, einen möglichst guten Gesundheitszustand für alle Völker zu erreichen, eine pragmatische Lösung finden sollte, bei der insbesondere auch die 23 Millionen Bewohner von Taiwan berücksichtigt werden. Dabei wäre die bestmögliche Lösung, einer taiwanesischen Health Entity den Status eines ständigen Beobachters bei den Sitzungen der Weltgesundheitsversammlung einzuräumen. ${ }^{26}$

$\mathrm{Zu}$ nennen sind insbesondere das Global Influenza Program (GIP), Global Early Warning and Response System (GLEWS), Global Influenza Surveillance Network (GISN) und die International Partnership on Avian and Pandemic Influenza (IPAPI).

26 Daten-Stand 2005.

Ein Beleg für diese Ansicht ist auch die Forderung eines derartigen Status durch das Europäische Parlament; vgl. Entschließung vom 18. Juli 1996 zur Rolle Taiwans in den internationalen Organisationen, AB1. C 261 vom 9.9.1996, S. 169; Entschließung zu Taiwan, B5-0347, 0356, 0372 und 0388/2000 vom 13. April 2000; Entschließung zu dem Beobachterstatus von Taiwan während der Jahrestagung der Weltgesundheitsversammlung (WHA) im Mai 2002 in Genf, B50130/2002 vom 14. März 2002; Entschließung zu Taiwan (56. Weltgesundheitsversammlung), B5-0224/2003 vom 15. Mai 2003. Der Vorschlag, die Frage des Beobachterstatus einer Einheit des taiwanesischen Gesundheitssystems bei der WHO auf die Tagesordnung zu setzen, wurde etwa bei der 56ten Sitzung der Weltgesundheitsversammlung vom 19.-28. Mai 2003 von Burkina-Faso, Costa-Rica, der Dominikanischen Republik, El Salvador, Gambia, Liberia, den 


\section{Verletzung von UN-Resolutionen oder der One China Policy?}

Schließlich bleibt die Frage, ob eine derartige Lösung gegen Resolutionen der Vereinten Nationen hinsichtlich der Vertretung Chinas in den internationalen Organisationen verstoßen würde oder mit der One China Policy zu vereinbaren wäre, nach der die Volksrepublik China und Taiwan einen einheitlichen Staat bilden. Bereits die Tatsache, dass es sich bei der Gewährung des Status eines ständigen Beobachters für eine Taiwan Health Entity nicht um eine Mitgliedschaft Taiwans in der WHO oder um eine offizielle Vertretung eines Staates welcher Art auch immer handelt, macht deutlich, dass kein Verstoß vorliegen würde. Eine lediglich technische Kooperation mit einer Einheit der Gesundheitsverwaltung Taiwans würde nicht die Frage des Status von Taiwan berühren. Eine Anerkennung Taiwans als Staat wäre weder Voraussetzung noch Rechtsfolge einer derartigen Kooperation, wie sich schon am Beispiel der derzeitigen sechs Beobachter zeigt. ${ }^{27}$

Auch schließen die Resolutionen der Vereinten Nationen einen solchen Beobachterstatus für eine Verwaltungseinheit nicht aus. Die Resolution 2768(XXVI) der Generalversammlung vom 25. Oktober 1971 legt nur fest, dass die Vertreter der Volksrepublik China als einzig legitime Vertreter Chinas bei den Vereinten Nationen anzuerkennen sind. Dem folgte die Weltgesundheitsversammlung in ihrer Entscheidung vom 10. Mai 1972. ${ }^{28}$ Bei der hier behandelten Frage handelt es sich indes nicht um die Vertretung Chinas bei der WHO, sondern lediglich um den ständigen Beobachterstatus einer Organisation der Gesundheitsfürsorge, die für die Bewohner Taiwans verantwortlich ist.

Dass eine derartige technische Kooperation die Frage des Status von Taiwan und die Vertretung Chinas bei internationalen Organisationen unberührt lässt, zeigen auch die bereits eingangs angeführten Beispiele des Straddling Stocks Übereinkommens und des darauf bezogenen regionalen Fischereiübereinkommens ${ }^{29}$. Beide im Rahmen des Seerechtsübereinkommens geschlossenen Übereinkommen gelten mutatis mutandis auch für andere

Marshall-Inseln, Nicaragua, Palau, Paraguay, Puerto-Rico, St. Kitts und Nevis, St. Vincent und die Grenadinen und den Vereinigten Staaten von Amerika unterstützt (WHA56/2003/REC/3). Er fand jedoch nicht die erforderliche Mehrheit. Im folgenden Jahr wurde erneut über einen derartigen Diskussionsvorschlag verhandelt. In Dokument EB114/2004/REC/1 des Exekutivrates ist die Diskussion über einen Beobachterstatus für Taiwan bei der Weltgesundheitsversammlung wiedergegeben. Jedoch entstand Streit darüber, ob die Einräumung eines derartige Status eine politische Frage oder eine Gesundheitsfrage sei und ob eine Diskussion darüber auf die Tagesordnung des Vorstands gesetzt werden sollte. Dieses wurde erneut abgelehnt. Vgl. auch die Dokumente WHA55/2002/REC/3, WHA/56/2003/REC/3 und WHA/58/2005/REC/3 der Weltgesundheitsversammlung.

27

Siehe oben Anm. 16.

28 Siehe die Entscheidung der Weltgesundheitsversammlung WHA25.1 vom 10. Mai 1972.

29

Siehe oben Anm. 4 und 5. 
fishing entities, deren Schiffe auf der Hohen See fischen. ${ }^{30}$ Bei der Verhandlung dieser Übereinkommen wurde ausdrücklich eine derartige Formulierung gewählt, um dem Status Chinas gerecht zu werden und zu gewährleisten, dass die Verpflichtungen auch für Taiwan gelten. ${ }^{31}$ Eine derartige fishing entity ist demnach nicht nur ein Beobachter, sondern sogar voll in die Durchführung der beiden Verträge integriert. Trotzdem waren sich die Vertragsstaaten gemeinsam mit China darin einig, dass dieses Konzept nicht im Widerspruch zur One China Policy oder der Vertretung Chinas bei den Vereinten Nationen steht. Ebenso wurde auch der WTO-Beitritt von „Chinese Taipei“, das unter anderem aus dem unabhängigen Zollgebiet von Taiwan besteht, als unabhängig von der Frage des Status Taiwans und der One China Policy bewertet. Auf eine ähnlich pragmatische Weise ist dem Primat der Funktionalität auch im Rahmen der WHO zur Geltung zu verhelfen. Die Frage des Gesundheitsschutzes darf nicht politisiert werden. Krankheiten machen keinen Halt vor Grenzen. Eine taiwanesische Health Entity ist daher von der WHO als ständiger Beobachter der Weltgesundheitsversammlung anzuerkennen.

\section{Zusammenfassung}

Der Status eines ständigen Beobachters ist nicht in der Satzung der WHO geregelt. Auch die Verfahrensordnung der Weltgesundheitsversammlung gibt insoweit lediglich gewisse Anhaltspunkte, verfügt jedoch über keine abschließende Regelung dieser Frage. Es hat sich in Bezug auf die derzeitigen sechs ständigen Beobachter jedoch eine Praxis herausgebildet, dass auf Bitte der Gesundheitsversammlung hin der Exekutivrat den Generaldirektor zur Erteilung des Beobachterstatus ermächtigt. Überdies steht der WHO das Recht zu, die für ihr Funktionieren erforderlichen Regelungen selbst zu treffen. Davon hat sie bisher schon wiederholt Gebrauch gemacht. In Bezug auf die Gewährung eines Beobachterstatus zugunsten der Gesundheitsverwaltung Taiwans in Form einer Taiwan Health Entity erscheint ein pragmatischer Ansatz geboten. Die umfassende Verpflichtung der WHO zur Erreichung des höchstmöglichen Gesundheitsstandards für alle Völker erfordert eine Berücksichtigung auch der 23 Millionen Menschen, die auf der Insel Taiwan beheimatet

30

So hat der Internationale Seegerichtshof in den Southern Bluefin Tuna Fällen die Geltung des Abkommens zum Schutz dieser Fische auch für „fishing entities“ gefordert, vgl. New Zealand v. Japan; Australia v. Japan, Provisional Measures, Order of 27 August 1999, Int. Legal Materials, Bd. 32, S. 1264, Nr. 90. In der Folge wurde Taiwan im August 2002 Mitglied der erweiterten Fischereikommission der Convention on the Conservation of Southern Bluefin Tuna von 1993; siehe Extended Commission for the Conservation of Southern Bluefin Tuna, Report of the Annual Meeting of the Commission, 15th to 18th October 2002, para. 2.

31 Vgl. D.H. Anderson, The Straddling Stocks Agreement of 1995 - an Initial Assessment, The International and Comparative Law Quaterly, Bd. 45 (1996), S. 463-475, 468; Bernard H.Oxman, Complementary Agreements and Compulsory Jurisdiction, The American Journal of International Law, Bd. 95 (2001), S. 277-312, 308 Fn. 142; Malcolm N. Shaw, International Law, 5. Aufl., Cambridge 2003, S. 558 Rn. 325. 
sind und in Gesundheitsfragen rein faktisch nicht effektiv durch die Volksrepublik China vertreten werden können. Ein derartiger Beobachterstatus ist unabhängig von der Frage des Status von Taiwan und verstößt daher auch nicht gegen die One China Policy. Eine Vertretung der Bevölkerung der Insel durch eine Taiwan Health Entity als ständiger Beobachter in der Weltgesundheitsversammlung der WHO ist daher zum Schutz ihrer Gesundheit und derjenigen anderer Völker unabdingbar und angesichts jederzeit möglicher grenzüberschreitender Epidemien dringend geboten. 